\title{
The histone methyltransferase SMYD2 is a novel therapeutic target for the induction of apoptosis in ovarian clear cell carcinoma cells
}

\author{
MACHIKO KOJIMA ${ }^{1}$, KENBUN SONE ${ }^{1}$, KATSUTOSHI ODA ${ }^{1}$, RYUJI HAMAMOTO ${ }^{2}$, SYUZO KANEKO $^{2}$, \\ SHINYA OKI ${ }^{1}$, ASAKO KUKITA ${ }^{1}$, AKIRA KAWATA ${ }^{1}$, HARUNORI HONJOH ${ }^{1}$, YOSHIKO KAWATA ${ }^{1}$, \\ TOMOKO KASHIYAMA $^{1}$, MASAKAZU SATO ${ }^{1}$, AYUMI TAGUCHI ${ }^{1}$, YUICHIRO MIYAMOTO $^{1}$, \\ MICHIHIRO TANIKAWA ${ }^{1}$, TETSUSHI TSURUGA ${ }^{1}$, KAZUNORI NAGASAKA ${ }^{3}$, \\ OSAMU WADA-HIRAIKE ${ }^{1}$, YUTAKA OSUGA ${ }^{1}$ and TOMOYUKI FUJII ${ }^{1}$ \\ ${ }^{1}$ Department of Obstetrics and Gynecology, Graduate School of Medicine, The University of Tokyo, Tokyo 113-8655; \\ ${ }^{2}$ Division of Molecular Modification and Cancer Biology, National Cancer Center Research Institute, Tokyo 104-0045; \\ ${ }^{3}$ Department of Obstetrics and Gynecology, Teikyo University School of Medicine, Tokyo 173-0003, Japan
}

Received January 5, 2020; Accepted June 29, 2020

DOI: $10.3892 / \mathrm{ol} .2020 .12014$

\begin{abstract}
Previous studies have suggested that histone methylation can modulate carcinogenesis and cancer progression. For instance, the histone methyltransferase SET and MYND domain containing 2 (SMYD2) is overexpressed in several types of cancer tissue. The aim of the present study was to determine whether SMYD2 could serve a therapeutic role in ovarian clear cell carcinoma (OCCC). Reverse transcription-quantitative PCR was used to examine SMYD2 expression in 23 clinical OCCC specimens. Moreover, OCCC cell proliferation and cell cycle progression were also examined following small interfering RNA-mediated SMYD2 silencing or treatment with a selective SMYD2 inhibitor. SMYD2 was significantly upregulated in clinical OCCC specimens, compared with normal ovarian tissue. In addition, SMYD2 knockdown decreased cell viability as determined via a Cell Counting Kit- 8 assay. Moreover, the proportion of cells in the sub- $\mathrm{G}_{1}$ phase increased following SMYD2 knockdown, suggesting increased apoptosis. Treatment with the SMYD2 inhibitor LLY-507 suppressed OCCC cell viability. These results suggested that SMYD2 could promote OCCC viability, and that SMYD2 inhibition
\end{abstract}

Correspondence to: Dr Kenbun Sone, Department of Obstetrics and Gynecology, Graduate School of Medicine, The University of Tokyo, 7-3-1 Hongo, Bunkyo-ku, Tokyo 113-8655, Japan E-mail: ksone5274@gmail.com

Abbreviations: OCCC, ovarian clear cell carcinoma; siRNA, small interfering RNA; NC, negative control; SMYD2, SET and MYND domain containing 2

Key words: histone methyltransferase, SMYD2, ovarian clear cell carcinoma, epigenetic modification, SMYD2 selective inhibitor induced apoptosis in these cells. Thus, SMYD2 inhibitors may represent a promising molecular targeted approach for OCCC treatment.

\section{Introduction}

Ovarian clear cell carcinoma (OCCC) was identified as a histopathological type of ovarian cancer by the World Health Organization in 1973 (1). OCCC accounts for 5-10\% of epithelial carcinomas in North America and for $25 \%$ of epithelial ovarian cancer cases in Japan (2). Furthermore, OCCC is chemoresistant, and thus patient prognosis is poorer, compared with serous or endometrioid carcinoma (2-4).

Epigenetic mechanisms, such as histone modification, regulate gene expression by altering chromatin structure $(5,6)$. Mutations in the chromatin remodeling gene AT rich interactive domain 1A (ARID1A) are present in $>50 \%$ of patients with OCCC (4). Histone methylation can include mono-, di- or trimethylation and is regulated by histone methyltransferases. Dysregulation of histone methylation has been implicated in cancer development and progression (6), and several types of histone methyltransferases are overexpressed in various types of cancer (6). For example, our previous study reported that histone methyltransferase suppressor of variegation 3-9 homolog 2 methylates histone $\mathrm{H} 2 \mathrm{AX}$ and regulates $\mathrm{H} 2 \mathrm{AX}$ phosphorylation during DNA repair (7). Other studies have indicated that histone methyltransferases could also represent potential therapeutic targets for $\operatorname{OCCC}(8,9)$. For instance, inhibition of enhancer of zeste 2 polycomb repressive complex 2 subunit (EZH2) has been shown to induce synthetic lethality in ARID1A-mutant OCCC cells, and sensitivity to EZH2 inhibitors is associated with ARID1A mutation status (8). Moreover, our previous study suggested that Wolf-Hirschhorn syndrome candidate 1 (WHSC1) was overexpressed and promoted cancer cell proliferation in OCCC. The expression of WHSC1 was attenuated via the knockdown or inhibition of EZH2 (9). EZH2 suppression attenuates cell proliferation 
and induces apoptosis, suggesting the possibility of a novel molecular target drug in endometrial cancer (10).

SET and MYND domain containing 2 (SMYD2) is a methyltransferase that methylates histones H3K4 and H3K36 (11). The SMYD2 protein can also methylate proteins other than histones, including $\mathrm{p} 53$, estrogen receptor $\alpha$ and $\mathrm{RB}$ transcriptional corepressor 1 (RB1), poly ADP ribose polymerase (PARP)-1, echinoderm microtubule associated protein like 4-ALK receptor tyrosine kinase fusion gene, heat shock protein 90 and $\beta$-catenin (12-17).

Our previous study suggested that SMYD2 was upregulated in clinical samples of high-grade serous ovarian cancer (HGSOC); additionally, SMYD2 suppression induced apoptosis in HGSOC cells (18). However, the role of SMYD2 in OCCC remains poorly understood. Therefore, the aim of the present study was to determine the role of SMYD2 in OCCC and evaluate its suitability as a potential therapeutic target. The expression of SMYD2 was measured in clinical OCCC specimens and normal ovarian tissues. Moreover, cell proliferation was examined in OCCC cell lines following SMYD2 gene silencing. The findings of the present study may provide insight into novel and effective therapeutic strategies for OCCC with.

\section{Materials and methods}

Clinical specimens and OCCC cell lines. Surgical specimens were collected from 23 patients with OCCC and 3 pathologically normal ovarian tissues removed for other diseases (Table SI). Samples were collected during surgery at The University of Tokyo Hospital between January 2006 and December 2016 after obtaining written patient consent and approval from the Human Genome, Gene Analysis Research Ethics Committee at the University of Tokyo (approval no. G0683-18). Tumor specimens were immediately snap-frozen in liquid nitrogen and stored at $-80^{\circ} \mathrm{C}$ prior to RNA extraction.

The OCCC cell lines OVISE (clone no. JCRB1043), OVTOKO (clone no. JCRB1048) and OVMANA (clone no. JCRB1045) were purchased from the Japanese Cancer Research Resources Bank. The TOV-21G OCCC cell line was purchased from American Type Culture Collection (clone no. ATCC ${ }^{\circledR}$ CRL-11730 ${ }^{\mathrm{TM}}$ ). OVISE, OVTOKO and TOV-21G cells were cultured in RPMI-1640 medium (FUJIFILM Wako Pure Chemical Corporation) with $10 \%$ FBS (Invitrogen; Thermo Fisher Scientific, Inc.) at $37^{\circ} \mathrm{C}$ with $5 \% \mathrm{CO}_{2}$. OVMANA cells were cultured under the same conditions using RPMI-1640 medium supplemented with $20 \%$ FBS. All cultures were confirmed Mycoplasma-free before and after the experiments using a MycoAlert ${ }^{\mathrm{TM}}$ Mycoplasma Detection kit (cat. no. LT07-218; Lonza Group, Ltd.).

Total RNA extraction and reverse transcription-quantitative PCR (RT-qPCR). Frozen specimens were embedded in Tissue-Tek ${ }^{\circledR}$ OCT $^{\text {тм }}$ compound (Sakura Finetek Japan Co., Ltd.), and 500- $\mu \mathrm{m}$ sections were cut using a CM1520 Cryostat (Leica Microsystems, Inc.). Then, 50-100 slices per specimen were placed in MagNA Lyser Green Beads and homogenized using a MagNA Lyser (both from Roche Diagnostics). Total RNA extraction from the supernatant (centrifugation at $8,000 \times \mathrm{g}$ for $1 \mathrm{~min}$ at $24^{\circ} \mathrm{C}$ ) was carried out using an RNeasy Mini kit (Qiagen, Inc.). Total RNA was reverse transcribed using ReverTra Ace- $\alpha$-(Toyobo Life Science) at $42^{\circ} \mathrm{C}$ for $20 \mathrm{~min}$ and $99^{\circ} \mathrm{C}$ for $5 \mathrm{~min}$. The mRNA expression levels of eight histone methyltransferases were measured using One-Step SYBR PrimeScript RT-PCR kit (Takara Bio, Inc.) on a Light Cycler instrument (Roche Diagnostics) using the following thermocycling conditions: Initial denaturation step at $95^{\circ} \mathrm{C}$ for $30 \mathrm{sec}$, followed by 45 cycles at $95^{\circ} \mathrm{C}$ for $10 \mathrm{sec}$, $55^{\circ} \mathrm{C}$ for $30 \mathrm{sec}$ and $72^{\circ} \mathrm{C} 30 \mathrm{sec}$, and a final extension step at $72^{\circ} \mathrm{C}$ for $10 \mathrm{~min}$. The primer sequences are given in Table SII. Gene expression was normalized to GAPDH mRNA levels, using the second derivative method (19).

Gene knockdown. SMYD2 knockdown was performed using small interfering (si)RNA (Sigma-Aldrich; Merck KGaA; Table SIII). OCCC cells (1x10\%/well in 6-well plates; $2 \times 10^{4} /$ well in 24-well plates) were transfected with $100 \mathrm{nM}$ siRNA using Lipofectamine ${ }^{\circledR}$ RNAi MAX transfection reagent (Invitrogen; Thermo Fisher Scientific, Inc.). The Mission siRNA Universal Negative Control (cat. no. SIC001; Sigma-Aldrich; Merck $\mathrm{KGaA}$ ) was used as a negative control (siNC). After $24 \mathrm{~h}$ from the start of cell culture, the cells were transfected with a specific siRNA and incubated for $48-96 \mathrm{~h}$ at $37^{\circ} \mathrm{C}$ before subsequent experiments.

Cell proliferation assay. Cell proliferation was assessed using the Cell Counting Kit-8 (CCK-8) method. OCCC cell lines were plated at a density of $2 \times 10^{4}$ cells/well in 24 -well plates and incubated for $24 \mathrm{~h}$. The cells were then transfected with siSMYD2 or siNC. CCK-8 (Dojindo Molecular Technologies, Inc.) was used 48-96 $\mathrm{h}$ following transfection according to the manufacturer's protocol, and the absorbance at $450 \mathrm{nM}$ was measured using an Epoch ${ }^{\mathrm{TM}}$ Microplate Spectrophotometer BioTek Instruments, Inc.). The experiment was repeated three times.

Western blotting. OCCC cells were plated at a density of $1 \times 10^{5}$ cells/wells in 6 -well plates and transfected with siSMYD2 or siNC. After transfection, cells were resuspended in lysis buffer $(50 \mathrm{mmol} / 1$ Tris- $\mathrm{HCl}, \mathrm{pH} \mathrm{7.5}$; $150 \mathrm{mmol} / 1$ sodium chloride; $5 \mathrm{mmol} / 1 \mathrm{EDTA} ; 2 \mathrm{mmol} / 1$ sodium orthovanadate; $10 \mathrm{mmol} / 1$ sodium fluoride). The lysate was centrifuged at $12,000 \mathrm{x}$ g for $20 \mathrm{~min}$ at $4^{\circ} \mathrm{C}$, and the protein concentration was then measured in the supernatant using a Bradford assay (Bio-Rad Laboratories, Inc.). Protein samples (30 $\mu \mathrm{g} /$ lane) were separated via SDS-PAGE(Any kD ${ }^{\mathrm{TM}}$ Mini-PROTEAN TGX Precast Gels; cat. no. 4569033; Bio-Rad Laboratories, Inc.) and transferred to poly vinylidene difluoride membranes (Bio-Rad Laboratories, Inc.) using the trans blot Turbo blotting system (Bio-Rad Laboratories, Inc.). The membranes were incubated with $5 \%$ skimmed milk in TBS-Tween $(0.1 \%$ Tween 20$)$ for $1 \mathrm{~h}$ with shaking at room temperature. After being blocked, the membranes were incubated overnight at $4^{\circ} \mathrm{C}$ with the following primary antibodies: Rabbit anti-SMYD2 (1:1,000; cat. no. 9734; Cell Signaling Technology, Inc.), rabbit anti-cleaved PARP (1:1,000; cat. no. 5625; Cell Signaling Technology, Inc.) and mouse anti- $\beta$-actin (1:3,000; cat. no. A2228; Sigma-Aldrich; Merck KGaA). Anti-rabbit IgG HRP-linked antibody (1:3,000; cat. no. 7074S; Cell Signaling Technology, Inc.) and anti-mouse IgG HRP-linked antibody $(1: 3,000$; 
A
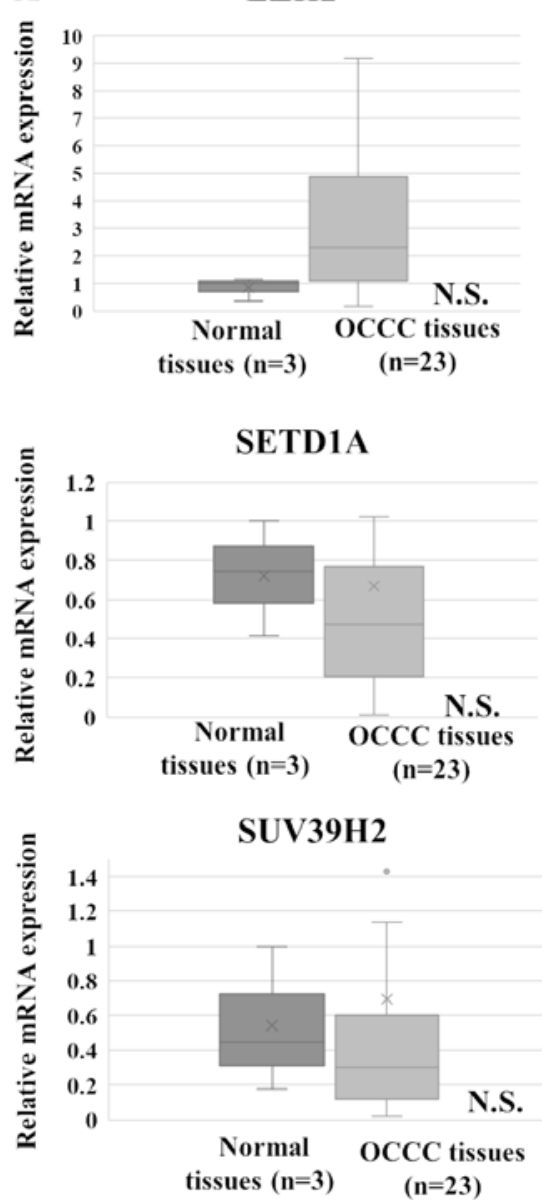

SMYD3

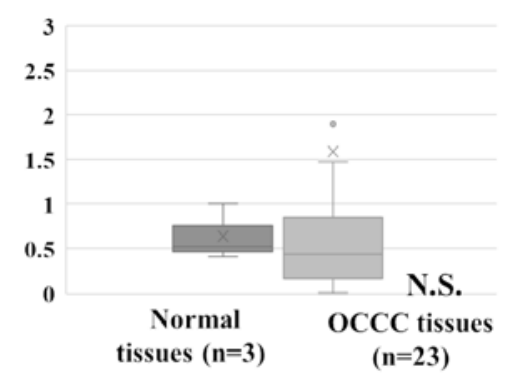

SETD8

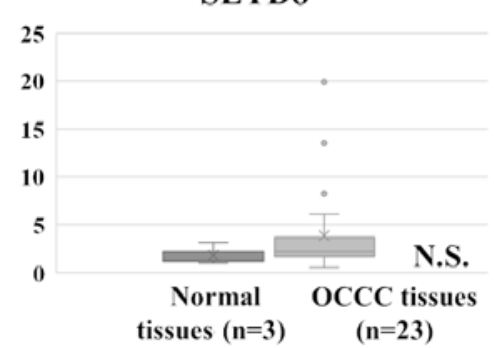

SMYD2

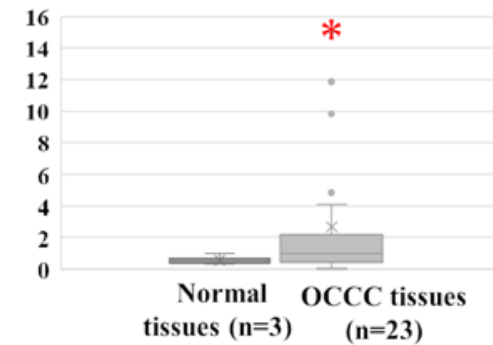

SETD7

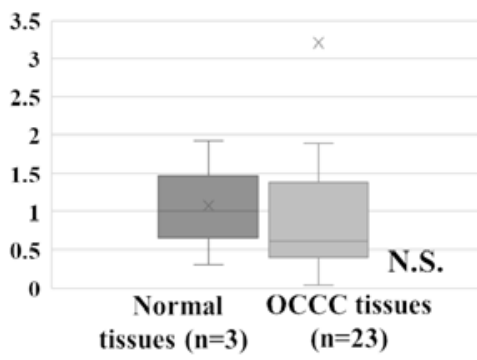

EHMT2
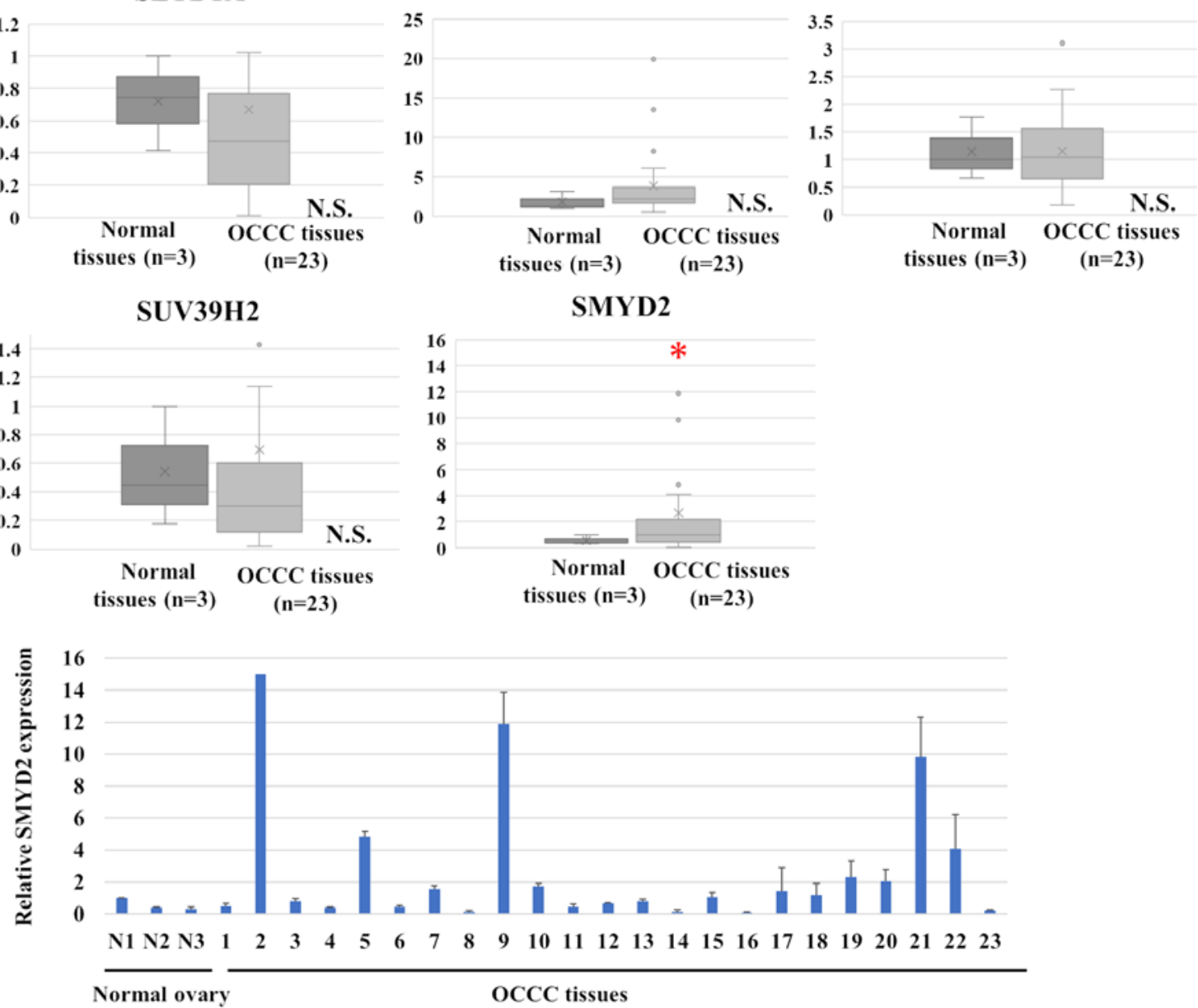

Figure 1. SMYD2 expression in OCCC. (A) Histone methyltransferase mRNA levels in OCCC clinical samples and normal ovarian samples. The data are displayed as box plots with the median, the average (represented by Xs), the maximum and minimum, the interquartile ranges and the outliers (represented by circles). (B) SMYD2 expression levels in individual study participants. The data is displayed as the mean \pm SD. *P<0.05. N.S., not significant; OCCC, ovarian clear cell carcinoma; EZH2, enhancer of zeste 2 polycomb repressive complex 2 subunit; SMYD, SET and MYND domain containing 2; SETD, SET domain containing lysine methyltransferase; SUV39H2, suppressor of variegation 3-9 homolog 2; EHMT2, euchromatic histone lysine methyltransferase 2.

cat. no. 7076S; Cell Signaling Technology, Inc.) were used as secondary antibodies and were incubated at room temperature for $1 \mathrm{~h}$ with shaking. Proteins were detected using ImageQuant LAS4000 (Cytiva) exposed under X-ray using an ECL select Western Blotting detection kit (Cytiva). Protein bands were quantified using ImageJ software (v1.52; National Institutes of Health).

Cell cycle analysis. OCCC cell lines were plated at a density $1 \times 10^{5}$ cells/well in 6 -well plates and cultured for $24 \mathrm{~h}$. The cells were then transfected with siSMYD2 or siNC. Following 48-72 $\mathrm{h}$ incubation, the cells were digested with trypsin, washed with PBS and then added to ice-cold $70 \%$ ethanol for $2 \mathrm{~h}$ at $4^{\circ} \mathrm{C}$. The samples were then incubated overnight at $4^{\circ} \mathrm{C}$, washed with PBS, then treated with $0.25 \mathrm{mg} / \mathrm{ml}$ RNase A (Sigma-Aldrich; Merck KGaA) at $37^{\circ} \mathrm{C}$ for $30 \mathrm{~min}$. Propidium iodide (final concentration, $50 \mu \mathrm{g} / \mathrm{ml}$ ) was then added to each sample and incubated for $30 \mathrm{~min}$ in the dark at $4^{\circ} \mathrm{C}$. Cell cycle progression was then evaluated using a FACSCalibur HG flow cytometer (BD Biosciences). The data were analyzed using the CellQuest Pro software (version 3.1; BD Biosciences).

$I C_{50}$ determination. The SMYD2 inhibitor LLY-507 was purchased from Selleck Chemicals (cat. no. S7575) and 
A

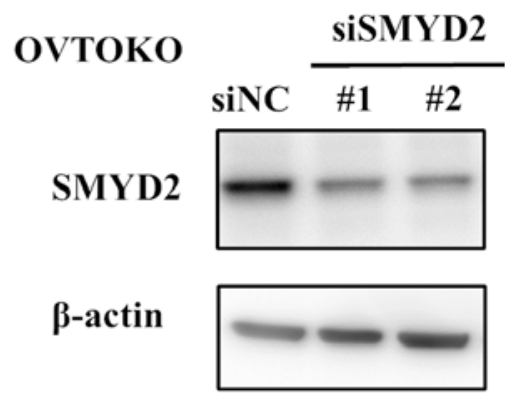

B OVTOKO

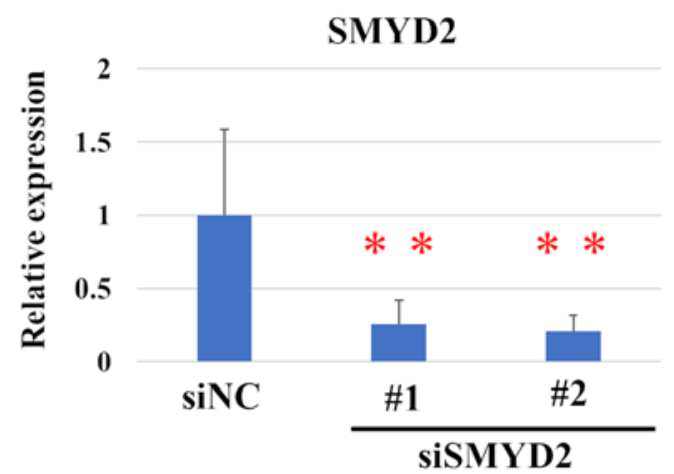

C OvToKo

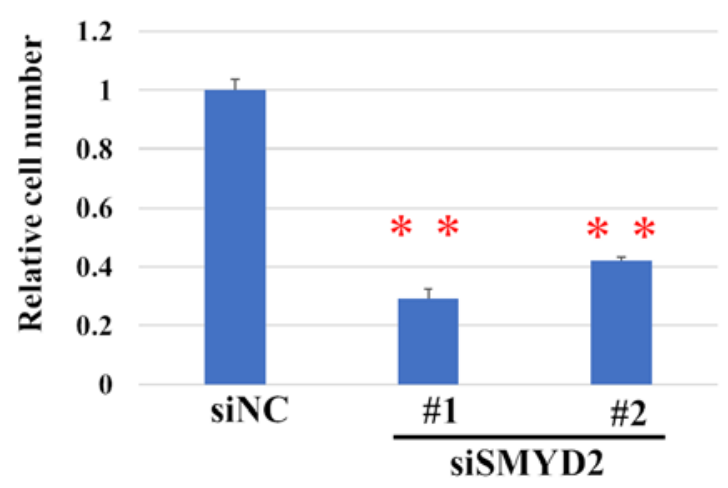

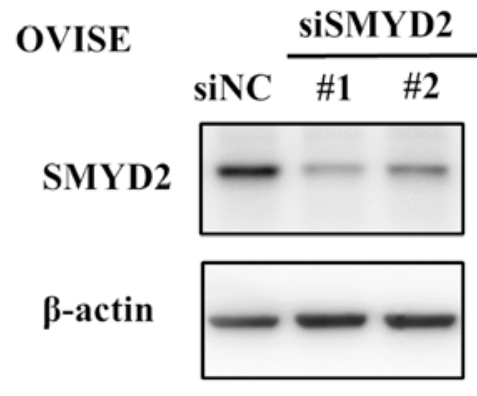

OVISE

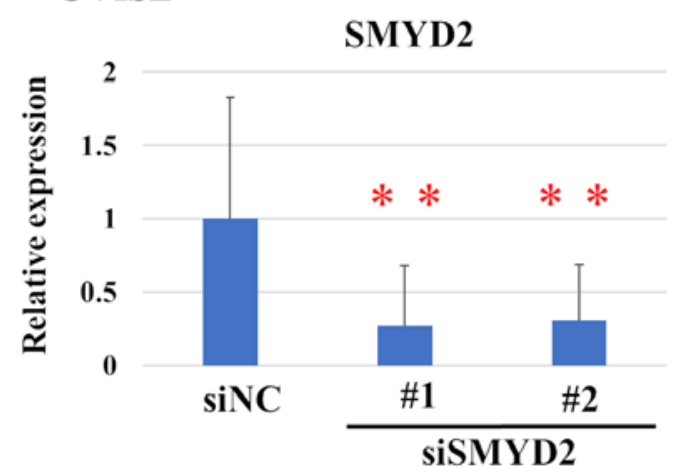

OVISE

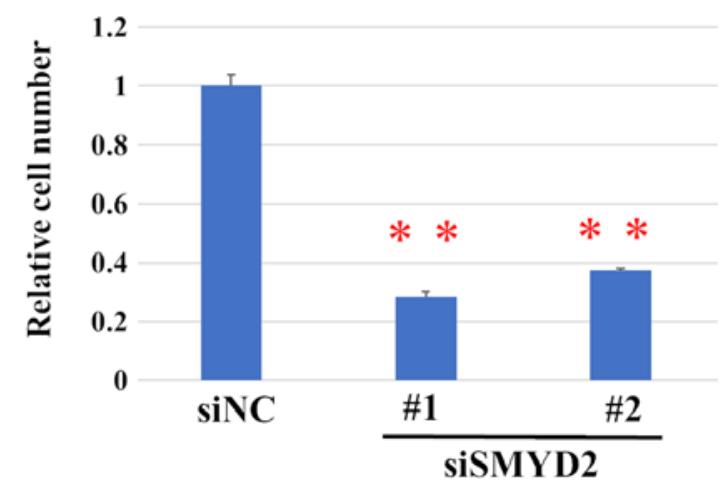

Figure 2. SMYD2 silencing attenuates proliferation of ovarian clear cell carcinoma cell lines. (A) SMYD2 knockdown was confirmed in OVTOKO and OVISE cells by western blotting following transfection with siSMYD2\#1 and \#2. (B) Semi-quantitative results of SMYD2 expression following siSMYD2 transfection. (C) SMYD2 knockdown significantly inhibits OVTOKO and OVISE cell proliferation. ${ }^{* *} \mathrm{P}<0.01$. SMYD, SET and MYND domain containing 2; si, small interfering; NC, negative control.

dissolved in $<0.1 \%$ DMSO for all experiments. OCCC cells were treated with LLY-507 at concentrations ranging from $1 \mathrm{nmol} / 1$ to $10 \mu \mathrm{mol} / 1$ or DMSO and cultured for 8 days. After adding CCK-8 solution, the number of viable cells was determined by measuring the absorbance at $450 \mathrm{~nm}$. Cell viability data in each experimental group were normalized the untreated cells. The $\mathrm{IC}_{50}$ was calculated using non-linear regression, as per the logistic curve equation. The cell viability, for both the control and the different LLY-507 treatment conditions, was plotted, and a logistic curve was drawn. The equation is as follows: $\mathrm{IC}_{50}=10^{\wedge}\left[\mathrm{LOG}(\mathrm{A} / \mathrm{B})^{*}(50-\mathrm{C}) /(\mathrm{D}-\mathrm{C})+\right.$ LOG(B)], where $\mathrm{A}$ is the higher concentration considering the two values that sandwich $50 \%$ cell viability, $\mathrm{B}$ is the lower concentration considering the same two values, $\mathrm{C}$ is the inhibition rate determined for $\mathrm{B}$, and $\mathrm{D}$ is the inhibition rate determined for $\mathrm{A}(20,21)$.
Statistical analysis. Statistical analysis was carried out using the JMP Pro software (version 14; SAS Institute, Inc.). The experiments were repeated three times, and the data are presented as the mean \pm SD. Comparisons between two groups were analyzed using F-test followed by unpaired Student's t-test. Multi-group comparisons were analyzed using one-way ANOVA and Tukey's post hoc test, and Kruskal-Wallis test was used for comparison between groups divided by TNM staging. $\mathrm{P}<0.05$ was considered to indicate a statistically significant difference.

\section{Results}

SMYD2 is overexpressed in OCCC cells. The expression of several histone methyltransferases reported to be overexpressed in other types of cancer $(7,13,20-25)$ were measured 

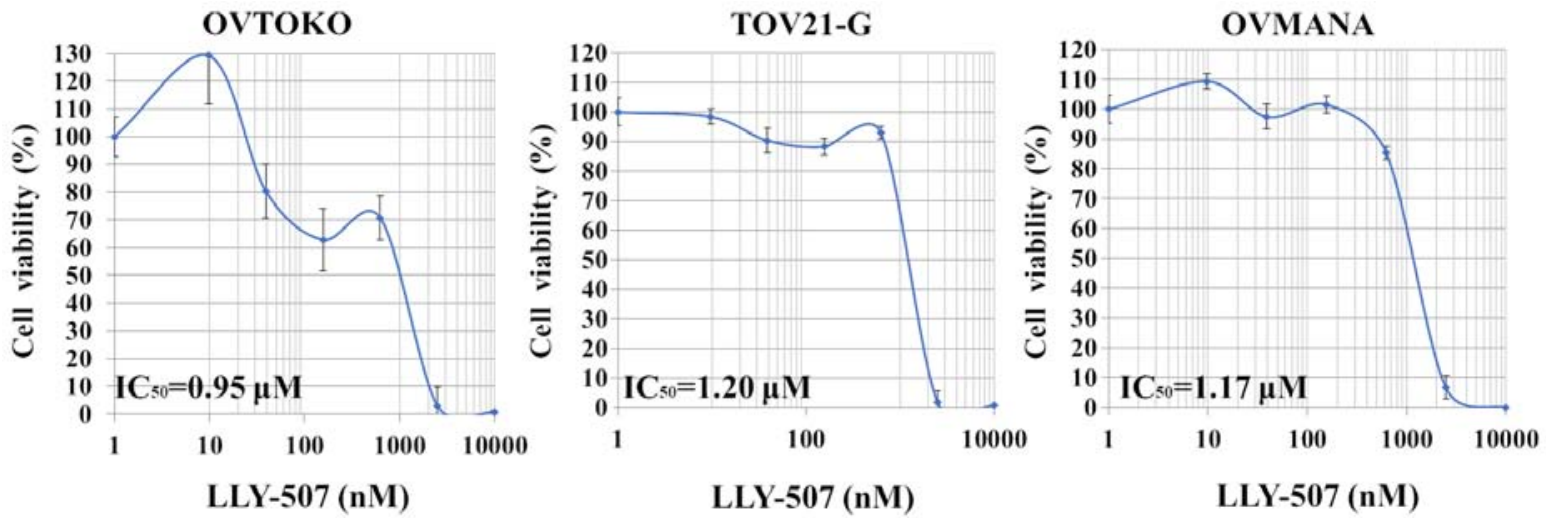

Figure 3. SMYD2 inhibition suppresses the proliferation of ovarian clear cell carcinoma cell lines. OVTOKO, TOV21-G and OVMANA cells were treated with LLY-507, a selective SMYD2 inhibitor, at concentrations ranging from 0.001-10 $\mu \mathrm{M}$. $\mathrm{IC}_{50}$ values are presented below the dose-response curve. SMYD, SET and MYND domain containing 2.

using RT-qPCR in order to evaluate their role in OCCC (Fig. 1). SMYD2 mRNA expression was significantly increased in OCCC tissues, compared with normal ovarian tissue $(\mathrm{P}=0.023)$. However, the expression of the eight remaining histone methyltransferases was similar in OCCC and normal ovarian tissue. The expression of SMYD2 in OCCC cell lines is also shown in Fig. S1. Only OVISE cells exhibited significantly higher SMYD2 expression compared with the other cell lines, but there was no difference in SMYD2 expression among the other cell lines.

The association between SMYD2 expression and age or TNM stage was also evaluated (Table SIV). Tumor tissues were divided according to SMYD2 expression, with expression $\geq$ median levels considered 'high', and expression < median classified as 'low'. The average age in both groups was 53 years. Moreover, SMYD2 expression was not associated with TNM stage. Thus, SMYD2 expression was not associated with these clinical variables.

SMYD2 promotes OCCC cell proliferation. The effect of SMYD2 silencing on cell proliferation was evaluated in the OVTOKO and OVISE cell lines, which have been previously used as representative OCCC cell lines $(22,23)$, using two specific siRNAs against SMYD2. OVTOKO and OVISE cells were transfected with siSMYD2\#1, siSMYD2\#2 or siNC SMYD2 expression was evaluated using western blot analysis (Fig. 2A). Transfection with either siMYD2\#1 or \#2 led to a significant reduction in the relative protein levels of SMYD2, compared with siNC (Fig. 2B).

Moreover, SMYD2 knockdown also significantly affected the viability of OCCC cells (Fig. 2C). Furthermore, the effect of a SMYD2-selective inhibitor, LLY-507, was also evaluated. Although the dose-response curve did not follow a strictly sigmoid trend, the viability of the OVTOKO, TOV-21G and OVMANA cell lines predominantly increased with the concentration of LLY-507 ( $\mathrm{IC}_{50}, 0.95,1.20$ and $1.17 \mu \mathrm{M}$, respectively; Fig. 3).

Suppression of SMYD2 induces apoptosis in OCCC cells. To examine the mechanism through which SMYD2 modulates the proliferation of OCCC cell lines, the levels of the apoptosis marker PARP were determined by western blot analysis (Fig. 4A). Cleaved PARP was detected in the SMYD2-knockdown group in OVTOKO and OVISE cell lines. Moreover, SMYD2 knockdown also significantly increased the proportion of OVTOKO and OVISE cells in the sub- $G_{1}$ phase of the cell cycle, compared with siNC, suggesting that SMYD2 silencing induced apoptosis (Figs. 4B and S2).

\section{Discussion}

SMYD2, which belongs to the group of transcriptional regulators containing SET and MYND domains, is responsible for complex transcriptional regulation through histone methylation and non-histone protein methylation $(24,25)$. Previous studies have suggested that SMYD2 is upregulated in several types of cancer, including pediatric acute lymphoblastic leukemia, gastric cancer and breast cancer (26-28). Additionally, previous studies have highlighted the role of SMYD2 in the regulation of cancer cell growth $(13,17)$. For example, SMYD2 is upregulated in gastric cancer and has important effects on cell proliferation (26). In the field of gynecologic cancer, an association between poor prognosis of cervical cancer and SMYD2 upregulation has been reported (29). Additionally, our previous study indicated that SMYD2 knockdown could suppress HGSOC cell proliferation and induce apoptosis (18). The findings of the present study are consistent with these previous reports. Higher SMYD2 expression was observed in OCCC tumor samples, compared with normal ovarian tissue. Furthermore, SMYD2 silencing inhibited OCCC cell proliferation and induced apoptosis. A SMYD2 selective inhibitor, LLY-507, also attenuated the proliferation of three OCCC cell lines.

It has been demonstrated that SMYD2 could attenuate the tumor suppressive function of p53 through methylation of Lys370 in the H1299 cell line, which is a human non-small cell lung carcinoma cell line (12). However, distinct mechanisms may account for HGSOC and OCCC cell apoptosis following SMYD2 knockdown. As $>90 \%$ of HGSOC cells have p53 mutations (18), it is possible that SMYD2 promotes apoptosis independently of p53 methylation. However, because p53 mutations are relatively infrequent in OCCC (30), SMYD2 knockdown could induce apoptosis by suppressing p53 
A
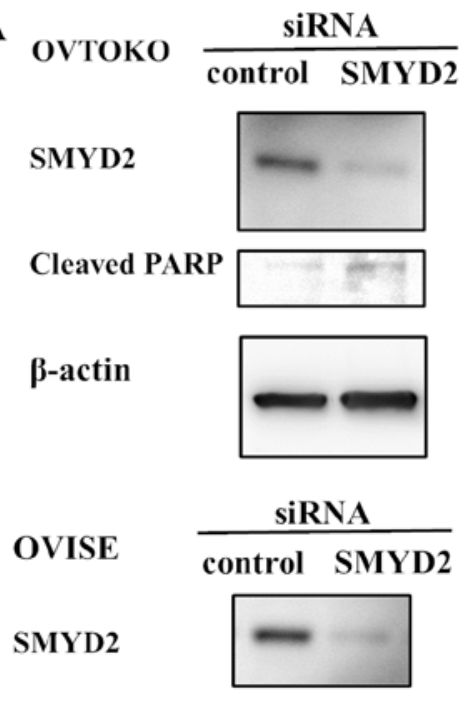

Cleaved PARP -

$\beta$-actin

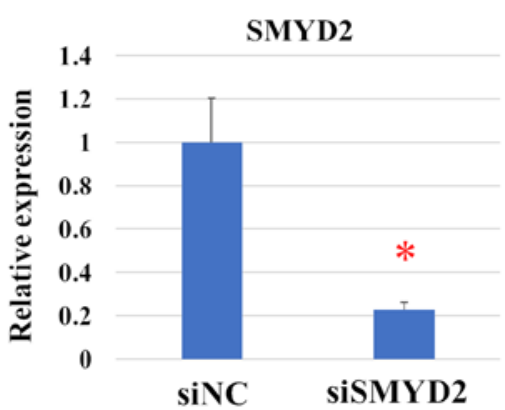

siNC siSMYD2

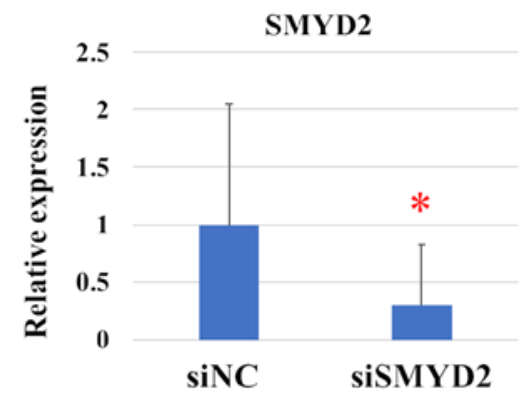

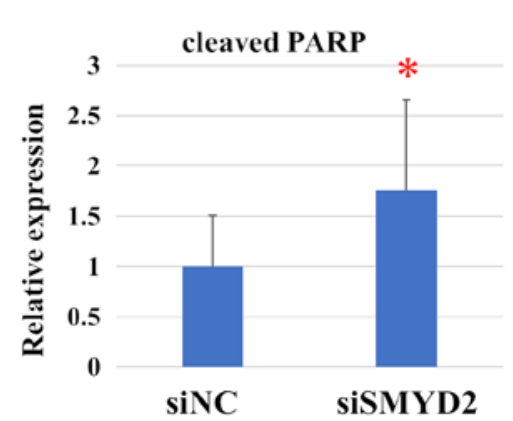

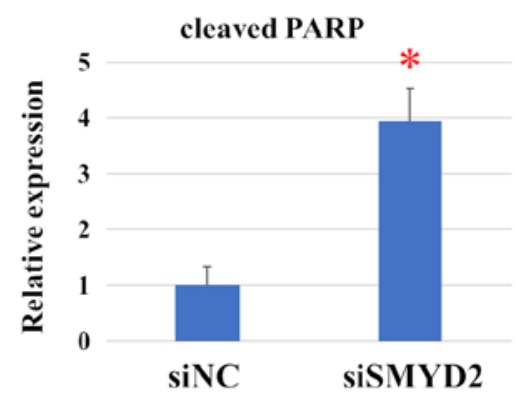

\section{B OVTOKO}

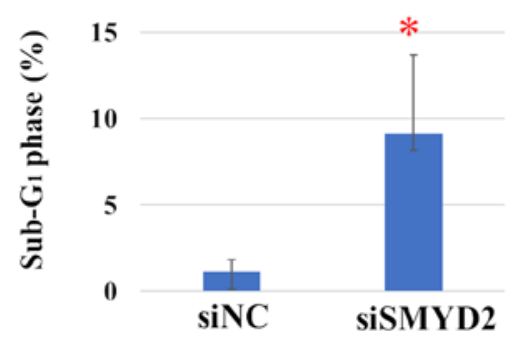

OVISE

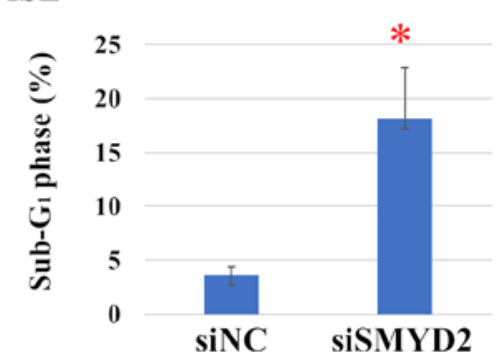

Figure 4. SMYD2 silencing induces apoptosis in OCCC cells. (A) SMYD2 and PARP protein expression in OCCC cell lines transfected with siSMYD2. (B) Frequency of cells in sub- $\mathrm{G}_{1}$ phase following siSMYD2 knockdown. An accumulation of cells in sub- $\mathrm{G}_{1}$ phase is indicative of increased apoptosis. "P<0.05. OCCC, ovarian clear cell carcinoma; SMYD2, SET and MYND domain containing 2; PARP, poly ADP ribose polymerase; si, small interfering; NC, negative control.

methylation. Moreover, other mechanisms might underlie the role of SMYD2 in carcinogenesis in OCCC, as SMDY2 can methylate several proteins other than histones (13-15). For instance, SMYD2 can methylate RB1 at Lys810, thereby modulating the cell cycle in bladder carcinoma (13). Furthermore, it has been reported that SMYD2 may affect the promotion of colorectal cancer metastasis by suppressing APC2 and activating the $\mathrm{Wnt} / \beta$-catenin signaling pathway (31). Additionally, it has been reported that SMYD2-mediated EML4-ALK methylation affects signaling pathways and proliferation of non-small cell lung cancer cells (16).

A previous study has indicated that LLY-507 induces apoptosis and suppresses HGSOC cell proliferation (18). Moreover, SMYD2 inhibitors have a long-term inhibitory effect on the proliferation of HGSOC cells in colony-formation assays (18). Another previous study has also indicated that LLY-507 suppresses the proliferation of esophageal, liver and breast cancer cells (32). The sensitivity to LLY-507 did not increase in a time-dependent manner in liver cancer cells, but breast cancer cells were 5 times more sensitive to LLY-507 after 7 days of treatment compared with after 3-4 days of treatment, suggesting that proliferation of breast cancer cells may be mediated, at least in part, by a SMYD2-dependent epigenetic mechanism (32). Consistent with these previous findings, the present study indicated that LLY-507 could inhibit the proliferation of OCCC cell lines. However, the antitumor effect of LLY-507 may differ in OCCC cell lines compared with HGSOC cells. Patients with OCCC rarely present p53 mutations, whereas $>90 \%$ of patients with HGSOC display p53 mutations (33). Therefore, it is speculated that the cytostatic effect of LLY-507 may be associated with the p53 signaling pathway in OCCC and alternative pathways, such as through histone modification in HGSOC. However, further studies are required to confirm this hypothesis. Moreover, there was no association between the cytostatic effect induced by SMYD2 suppression and its expression in OCCC cell lines.

The present study has several limitations. As all experiments were carried out in vitro, extensive in vivo validation is required to ascertain whether SMYD2 might serve as a potential therapeutic target in OCCC. Moreover, the mechanism of 
action underlying the effect of SMYD2 on cell proliferation and apoptosis, including the potential role of p53 methylation, has not been addressed. Lastly, endometriosis is reportedly a precursor of OCCC (34), and therefore analysis of SMYD2 expression in OCCC should be compared with normal ovarian tissues as well as with tissues of endometriosis in the future. Nevertheless, the present findings suggest that SMYD2 increases the proliferation of OCCC cells in vitro, suggesting a potential therapeutic avenue for SMYD2 inhibition in the treatment of OCCC.

\section{Acknowledgements}

Not applicable.

\section{Funding}

This work was financially supported by a Grant-in-Aid for Scientific Research (grant nos. 18K09249, 15K10705 and 17K11268) and a Grant-in-Aid for Research Activity Start-up (grant no. 15H06173) from The Ministry of Education, Culture, Sports, Science and Technology of Japan. This research was also supported by the Project for Cancer Research and Therapeutic Evolution from The Japan Agency for Medical Research and Development (grant no. 17K11268).

\section{Availability of data and materials}

All data generated and analyzed during this study are included in this published article.

\section{Authors' contributions}

MK, KS and KO conceived and designed the study. MK, KS, $\mathrm{RH}$ and SK designed the experiments. All experiments were performed by MK. The data were analyzed and interpreted by SO, AKu, AKa, HH, YK, TK, MS, AT, MT, YM, TT, KN, $\mathrm{OH}, \mathrm{YO}$ and TF. MK and $\mathrm{KS}$ prepared the manuscript and figures. MK, KS, KO, RH, SK, TT, YO and TF reviewed and revised the manuscript for important intellectual content. Technical and material support was provided by $\mathrm{SO}, \mathrm{AKu}$, $\mathrm{HH}$ and YK. All the authors read and approved the final manuscript.

\section{Ethics approval and consent to participate}

The study protocol was approved by the Human Genome, Gene Analysis Research Ethics Committee at the University of Tokyo. Written informed consent was obtained from the patients for the research use of the tumor specimens and their clinical data as a whole.

\section{Patient consent for publication}

Not applicable.

\section{Competing interests}

KS has a research grant from Daiichi-Sankyo Co., Ltd. KO has a research grant from Daiichi-Sankyo Co., Ltd. and
AstraZeneca Plc. and received a lecture fee from Chugai Pharmaceutical Co., Ltd. and AstraZeneca Plc. All other authors declare that they have no competing interests.

\section{References}

1. Serov SF Scully R and Sobin LH: Histologic typing of ovarian tumors. Geneva: World Health Organization, 1973.

2. Mabuchi S, Sugiyama T and Kimura T: Clear cell carcinoma of the ovary: Molecular insights and future therapeutic perspectives. J Gynecol Oncol 27: e31,2016.

3. Crotzer DR, Sun CC, Coleman RL, Wolf JK, Levenback CF and Gershenson DM: Lack of effective systemic therapy for recurrent clear cell carcinoma of the ovary. Gynecol Oncol 105: 404-408, 2007.

4. Takano M, Sugiyama T, Yaegashi N, Sakuma M, Suzuki M, Saga Y, Kuzuya K, Kigawa J, Shimada M, Tsuda H, et al: Low response rate of second-line chemotherapy for recurrent or refractory clear cell carcinoma of the ovary: A retrospective Japan Clear Cell Carcinoma Study. Int J Gynecol Cancer 18: 937-942, 2008.

5. Varier RA and Timmers HT: Histone lysine methylation and demethylation pathways in cancer. Biochim Biophys Acta 1815: 75-89, 2011.

6. Hamamoto R, Saloura V and Nakamura Y: Critical roles of non-histone protein lysine methylation in human tumorigenesis. Nat Rev Cancer 15: 110-124, 2015.

7. Sone K, Piao L, Nakakido M, Ueda K, Jenuwein T, Nakamura Y and Hamamoto R: Critical role of lysine 134 methylation on histone $\mathrm{H} 2 \mathrm{AX}$ for gamma-H2AX production and DNA repair. Nat Commun 5: 5691, 2014.

8. Bitler BG, Aird KM, Garipov A, Li H, Amatangelo M, Kossenkov AV, Schultz DC, Liu Q, Shih IeM, Conejo-Garcia JR, et al: Synthetic lethality by targeting EZH2 methyltransferase activity in ARID1A-mutated cancers. Nat Med 21: 231-238, 2015.

9. Kojima M, Sone K, Oda K, Hamamoto R, Kaneko S, Oki S, Kukita A, Machino H, Honjoh H, Kawata Y, et al: The histone methyltransferase WHSC1 is regulated by EZH2 and is important for ovarian clear cell carcinoma cell proliferation. BMC Cancer 19: 455, 2019.

10. Oki S, Sone K, Oda K, Hamamoto R, Ikemura M, Maeda D, Takeuchi M, Tanikawa M, Mori-Uchino M, Nagasaka K, et al: Oncogenic histone methyltransferase EZH2: A novel prognostic marker with therapeutic potential in endometrial cancer. Oncotarget 8: 40402-40411, 2017.

11. Brown MA, Sims RJ III, Gottlieb PD and Tucker PW: Identification and characterization of Smyd2: A split SET/MYND domain-containing histone H3 lysine 36-specific methyltransferase that interacts with the Sin3 histone deacetylase complex. Mol Cancer 5: 26, 2006

12. Huang J, Perez-Burgos L, Placek BJ, Sengupta R, Richter M, Dorsey JA, Kubicek S, Opravil S, Jenuwein T and Berger SL: Repression of p53 activity by Smyd2-mediated methylation. Nature 444: 629-632, 2006.

13. Cho HS, Hayami S, Toyokawa G, Maejima K, Yamane Y, Suzuki T, Dohmae N, Kogure M, Kang D, Neal DE, et al: RB1 methylation by SMYD2 enhances cell cycle progression through an increase of RB1 phosphorylation. Neoplasia 14: 476-486, 2012.

14. Piao L, Kang D, Suzuki T, Masuda A, Dohmae N, Nakamura Y and Hamamoto R: The histone methyltransferase SMYD2 methylates PARP1 and promotes poly(ADP-ribosyl)ation activity in cancer cells. Neoplasia 16: 257-264, 264.e2, 2014.

15. Hamamoto R, Toyokawa G, Nakakido M, Ueda K and Nakamura Y: SMYD2-dependent HSP90 methylation promotes cancer cell proliferation by regulating the chaperone complex formation. Cancer Lett 351: 126-133, 2014.

16. Wang R, Deng X, Yoshioka Y, Vougiouklakis T, Park JH, Suzuki T, Dohmae N, Ueda K, Hamamoto R and Nakamura Y: Effects of SMYD2-mediated EML4-ALK methylation on the signaling pathway and growth in non-small-cell lung cancer cells. Cancer Sci 108: 1203-1209, 2017.

17. Deng X, Hamamoto R, Vougiouklakis T, Wang R, Yoshioka Y, Suzuki T, Dohmae N, Matsuo Y, Park JH and Nakamura Y: Critical roles of SMYD2-mediated $\beta$-catenin methylation for nuclear translocation and activation of Wnt signaling. Oncotarget 8: 55837-55847, 2017. 
18. Kukita A, Sone K, Oda K, Hamamoto R, Kaneko S, Komatsu M, Wada M, Honjoh H, Kawata Y, Kojima M, et al: Histone methyltransferase SMYD2 selective inhibitor LLY-507 in combination with poly ADP ribose polymerase inhibitor has therapeutic potential against high-grade serous ovarian carcinomas. Biochem Biophys Res Commun 513: 340-346, 2019.

19. Livak KJ and Schmittgen TD: Analysis of relative gene expression data using real-time quantitative PCR and the 2(-Delta Delta C(T)) method. Methods 25: 402-408, 2001.

20. Yokogi S, Tsubota T, Kanki K, Azumi J, Itaba N, Oka H, Morimoto M, Ryoke K and Shiota G: Wnt/Beta-catenin signal inhibitor HC-1 sensitizes oral squamous cell carcinoma cells to 5 -fluorouracil through reduction of CD44-positive population. Yonago Acta Med 59: 93-99, 2016.

21. Lyles RH, Poindexter C, Evans A, Brown M and Cooper CR: Nonlinear model-based estimates of IC(50) for studies involving continuous therapeutic dose-response data. Contemp Clin Trials 29: 878-886, 2008

22. Price C, Gill S, Ho ZV, Davidson SM, Merkel E, McFarland JM, Leung L, Tang A, Kost-Alimova M, Tsherniak A, et al: Genome-wide interrogation of human cancers identifies EGLN1 dependency in clear cell ovarian cancers. Cancer Res 79: 2564-2579, 2019.

23. Anglesio MS, Wiegand KC, Melnyk N, Chow C, Salamanca C, Prentice LM, Senz J, Yang W, Spillman MA, Cochrane DR, et al: Type-specific cell line models for type-specific ovarian cancer research. PLoS One 8: e72162, 2013.

24. Singh PK: Histone methyl transferases: A class of epigenetic opportunities to counter uncontrolled cell proliferation. Eur J Med Chem 166: 351-368, 2019.

25. Abu-Farha M, Lambert JP, Al-Madhoun AS, Elisma F, Skerjanc IS and Figeys D: The tale of two domains: Proteomics and genomics analysis of SMYD2, a new histone methyltransferase. Mol Cell Proteomics 7: 560-572, 2008.

26. Komatsu S, Ichikawa D, Hirajima S, Nagata H, Nishimura Y, Kawaguchi T,Miyamae M, Okajima W, Ohashi T, Konishi H, et al: Overexpression of SMYD2 contributes to malignant outcome in gastric cancer. Br J Cancer 112: 357-364, 2015.
27. Li LX, Zhou JX, Calvet JP, Godwin AK, Jensen RA and Li X: Lysine methyltransferase SMYD2 promotes triple negative breast cancer progression. Cell Death Dis 9: 326, 2018.

28. Sakamoto LH, Andrade RV, Felipe MS, Motoyama AB and Pittella Silva F: SMYD2 is highly expressed in pediatric acute lymphoblastic leukemia and constitutes a bad prognostic factor. Leuk Res 38: 496-502, 2014.

29. Sun JJ, Li HL, Ma H, Shi Y, Yin LR and Guo SJ: SMYD2 promotes cervical cancer growth by stimulating cell proliferation. Cell Biosci 9: 75, 2019.

30. Ho ES, Lai CR, Hsieh YT, Chen JT, Lin AJ, Hung MH and Liu FS: p53 mutation is infrequent in clear cell carcinoma of the ovary. Gynecol Oncol 80: 189-193, 2001.

31. Meng F, Liu X, Lin C, Xu L, Liu J, Zhang P, Zhang X, Song J, Yan Y, Ren Z and Zhang Y: SMYD2 suppresses APC2 expression to activate the $\mathrm{Wnt} / \beta$-catenin pathway and promotes epithelial-mesenchymal transition in colorectal cancer. Am J Cancer Res 10: 997-1011, 2020.

32. Nguyen H, Allali-Hassani A, Antonysamy S, Chang S, Chen LH, Curtis C, Emtage S, Fan L, Gheyi T, Li F, et al: LLY-507, a cell-active, potent, and selective inhibitor of protein-lysine methyltransferase SMYD2. J Biol Chem 290: 13641-13653, 2015.

33. Cole AJ, Dwight T, Gill AJ, Dickson KA, Zhu Y, Clarkson A, Gard GB, Maidens J, Valmadre S, Clifton-Bligh R and Marsh DJ: Assessing mutant p53 in primary high-grade serous ovarian cancer using immunohistochemistry and massively parallel sequencing. Sci Rep 6: 26191, 2016.

34. Ishibashi H, Takano M, Miyamoto M, Soyama H, Matsuura H, Aoyama T, Yoshikawa T, Kato K, Tsuda H and Furuya K: Role of endometriosis as a prognostic factor for post-progression survival in ovarian clear cell carcinoma. Mol Clin Oncol 7: 1027-1031, 2017.

(i) (i) This work is licensed under a Creative Commons

c) Attribution-NonCommercial-NoDerivatives 4.0 International (CC BY-NC-ND 4.0) License. 